schen Maßnahmen, doch die Umsetzung der Reformen, der damit vollzogene institutionelle Wandel und ihr exklusiver bzw. inklusiver Charakter ist stark von internen Rahmenbedingungen (Kräftekonstellationen) motiviert.

Trotz der insgesamt gelungenen Konstruktion und Durchführung der Vergleichsstudie sind auch Kritikpunkte anzuführen. Während die konzeptionelle Fassung der Dezentralisierung überzeugt, gilt dies nicht im gleichen Maße für den Bereich der Legitimation. Die zwei verwendeten Kategorien "Herrschaftssicherung" und "eliteninterne Konfliktregulierung" sind analytisch nicht trennscharf gehalten, was sich auch in der empirischen Anwendung zeigt. Nicht näher reflektiert wird der für die Argumentation nicht unbedeutende Begriff des Einflusses. Hilfreich für die Argumentation der Zusammenhänge der Dezentralisierungsmaßnahmen als abhängige und unabhängige Variable wäre eine Überführung der Diskussion der drei aufgegriffenen Theoriestränge in allgemeine Arbeitshypothesen gewesen. Schließlich ist in einigen Passagen eine stärkere empirische Fundierung der Befunde zu wünschen. Dies betrifft Aussagen zur Legitimationsentwicklung und die für beide Länder nicht immer parallel vorhandenen statistischen Informationen. Doch diese Kritikpunkte treten hinter die Leistung der Studie zurück, die im Unterschied zur oftmals diffusen Debatte über lateinamerikanische Dezentralisierungsprozesse sinnvolle Klassifikationsvorschläge unterbreitet und anwendet. Darauf aufbauend identifiziert die Untersuchung zugleich Chancen und Gefahren für eine gelungene Entwicklung, auf deren Grundlage der Autor zum einen den Bedarf an einer konsistenten Dezentralisierung anmahnt und zum anderen vor dem Hintergrund behinderter odẹ unzureichend realisierter Reformstrategien, die zu neuen oligopolistischen Marktstrukturen führen können, vor einer Dezentralisierungseuphoric warnt.

Hans-Joachim Lauth

\title{
Ivo Joswig
}

Die implied powers-Lehre im amerikanischen Verfassungsrecht

Eine Analyse anhand der Rechtsprechung des U.S. Supreme Court

Europäische Hochschulschriften, Reihe II, Rechtswissenschaft, Band 1979

Peter Lang Verlag, Frankfurt a.M., 1996, 241 S., DM 79,--

In Verfassungsordnungen mit geteilter Staatsgewalt ist die Frage der Kompetenzverteilung von herausragender Bedeutung. Das gilt im föderalen Verfassungssystem sowohl in vertikaler Hinsicht im Verhältnis zwischen Gliedstaaten und Gesamtstaat als auch in horizontaler Hinsicht im Verhältnis von Legislative, Exekutive und Judikative. Mit der implied powers-Lehre werden Reichweite und Grenzen von Kompetenzen bestimmt, indem von einer in der Verfassungsordnung ausdrücklich enthaltenen Aufgabennorm auf die Kompetenz zur Erfüllung dieser Aufgabe geschlossen wird. Die so bestimmten Kompetenzen sind 
nach dieser Lehre von der Verfassung bereits einbegriffen (implied) und werden im konkreten Anwendungsfall durch einen logischen Schluß ermittelt.

Die von Heun betreute Dissertation von Ivo Joswig untersucht Entwicklung und Anwendungsbereiche der implied powers-Lehre, die auch im Völker- und Europarecht Geltung beansprucht, im U.S.-amerikanischen Verfassungsrecht. Dort ist diese Lehre in der Verfassungsdiskussion des ausgehenden 18. Jahrhundert und bis heute in der Rechtsprechung des U.S. Supreme Court entwickelt worden, was bereits eine vertiefte Beschäftigung mit dieser Thematik verdient.

Die Arbeit ist in vier Teile gegliedert. Joswig beginnt im ersten Teil mit einem historischen Überblick der Verfassungsentwicklung bis zum leading case der implied powers-Lehre des Supreme Court McCulloch v. Maryland aus dem Jahre 1819 (S. 1-48). Das Verhältnis von Einzelstaaten und Gesamtstaat war von Anfang an der Hauptanwendungsbereich der implied powers-Lehre. Waren die Articles of Confederation von 1777 als erster Gesamtverfassung der Vereinigten Staaten noch von einer stark souveränen Stellung der Einzelstaaten geprägt, enthielt die Verfassung von 1787 bereits die Normen, die für die Entwicklung der implied powers-Lehre wegweisend sein sollten. In Art. 1, sec. 8, cl. 1-17 sind bestimmte Gesetzgebungskompetenzen des Bundes enthalten. $\mathrm{Cl}$. 18, die sog. necessary and proper clause, bestimmt daneben, daß der Kongreß die Befugnis haben soll, "... to make all Laws which shall be necessary and proper for carrying into Execution the foregoing Powers, and all other Powers vested by this Constitution in the Government of the United States, or in any Department or Office thereof". Bereits in dieser Formulierung wird deutlich, daß die cl. 1-17 nicht nur bestimmte Kompetenzen aufzählen, sondern darüber hinaus auch Zielsetzungen für weitere Kompetenzen gemäß der cl. 18 enthalten. Schließlich ist noch auf das 10. Amendment hinzuweisen, das bestimmt: "The powers not delegated to the Unites States by the Constitution, nor prohibited by it to the State, are reserved to the States respectively, or to the people". Schon während der Ratifikationsdebatte entbrannte heftiger Streit darüber, wie die necessary and proper clause zu interpretieren sei. Deutlich wurden die Positionen 1791 im Zusammenhang mit der Errichtung einer Nationalbank durch ein Bundesgesetz (S. 22-29). Nach der unter anderem von Jefferson vertretenen restriktiveren Interpretation fehlte es an einer entsprechenden Bundeskompetenz, weil es sich zwar um eine zweckmäßige, aber nicht wirklich notwendige Maßnahme zur Erreichung der in Art. 1, sec. 8, cl. 1-17 enumerierten Zielsetzungen handelte. Die extensivere, unter anderem von Hamilton vertretene Auffassung nahm hingegen eine sog. implied power des Bundes an und begründete dies mit einer "natürlichen Verbindung" zwischen der in Frage stehenden Maßnahme und einer der ausdrücklich in der Verfassung genannten Zielsetzungen. Lediglich "great independent substantive things", die keine sachliche Verbindung zu einer der enumerierten Zielsetzungen aufwiesen, konnten danach von einer Bundeskompetenz nicht erfaßt werden. Präsident Washington folgte der extensiveren Auffassung und unterzeichnete das Nationalbank-Gesetz. In McCulloch v. Maryland schloß sich der Supreme Court ebenfalls dieser Ansicht an. Dem Kongreß wurde ferner ein legislatives Ermessen hinsichtlich der Frage eingeräumt, wann er bestimmte Maßnahmen zur 
Erreichung der in cl. 1-17 genannten Zielsetzungen für erforderlich erachtete. Damit ging eine Rücknahme der verfassungsgerichtlichen Kontrollbefugnis einher, da der Supreme Court es nicht als seine Aufgabe ansah, Bundesgesetze auf ihre Zweckmäßigkeit hin zu überprüfen. Der historische Überblick ist knapp und übersichtlich gehalten. Manchmal wird dem Leser leider das Nachvollziehen der Problematik dadurch erschwert, daß in der Darstellung der konkreten Anwendungsfälle nicht ausdrücklich der in der Verfassung in cl. 1-17 konkret geregelte Sachbereich genannt wird, um das "interpretatorische Ausmaß" der implied powers-Lehre zu ermessen.

Im zweiten Teil stellt Joswig die Rechtsprechung des Supreme Court zur Anwendung der implied powers-Lehre im Verhältnis von Einzelstaaten und Gesamtstaat am Beispiel der umfassenden Bundesgesetzgebung für Handel und Wirtschaftsregulierung dar (S. 49-141). Im Mittelpunkt stehen dabei zum einen die Anforderungen, die entsprechend dem dargestellten Grundverständnis der implied powers-Lehre an die Zweck-Mittel-Relation zwischen verfassungsrechtlichen Zielsetzungen in cl. 1-17 und den in Frage stehenden Maßnahmen zu stellen sind, und zum anderen die Frage nach der verfassungsgerichtlichen Kontrollbefugnis. Nachdem der Supreme Court anfangs einen formalen Ansatz zur Ermittlung der geforderten Zweck-Mittel-Relation gefordert hatte (direct and indirect effect), setzte sich im Kontext der New Deal-Gesetzgebung ab 1937 eine stärker ökonomische Betrachtungsweise durch, und eine "close and substantial relationship" wird seitdem für die Kompetenzbegründung für ausreichend erachtet (erster Prüfungsschritt). Sowohl tatsächliche wirtschaftliche Zusammenhänge als auch das Anknüpfen an rein lokale private Aktivitäten können nunmehr diese Zweck-Mittel-Relation begründen. Eine "trivial relationship" im Sinne eines lediglich geringfügigen Zusammenhangs soll hingegen nicht genügen. In einem zweiten Prüfungsschritt steht die Frage im Mittelpunkt, inwieweit es dem Supreme Court zukommen soll, das Vorhandensein einer close and substantial relationship festzustellen. Nach anfänglich strikterer Kontrolle räumt der Supreme Court dem Kongreß seit den 60er Jahren des 20. Jahrhunderts ein umfassendes legislatives Ermessen im Sinne eines Beurteilungsspielraums ein und unterzieht seitdem Kongreßgesetze nur einem rational basis test, einer mit der Willkürprüfung des Bundesverfassungsgerichts vergleichbaren Vertretbarkeitskontrolle. Vom Kongreß wird dabei verlangt, daß er selbst umfangreiche Ermittlungen zur Feststellung der geforderten Zweck-Mittel-Relation anstellt. Das kann sowohl im Gesetz selbst (z.B. der Präambel) als auch in sonstigen Gesetzgebungsmaterialien erfolgen. Ist dies geschehen, geht der Supreme Court im Sinne einer grundsätzlichen Vermutung von der Verfassungsmäßigkeit des Bundesgesetzes aus. Joswig stellt schließlich noch die Rechtsprechung zum 10. Amendment dar, die von einer wechselhaften Entwicklung gekennzeichnet ist. Sollte ihm mal nur deklaratorische Bedeutung zukommen, weil die Frage der Gesetzgebungsbefugnisse in Art. 1, sec. 8, cl. 1-18 abschließend geregelt sei, wurde ihm auch die Einräumung eines unantastbaren Kernbereiches einzelstaatlicher Kompetenzen und damit eine eigenständige Grenze für die Gesetzgebungstätigkeit des Bundes entnommen. Joswig konstatiert am Ende des zweiten Teils eine starke Zurückhaltung in der heutigen Rechtsprechung des Supreme Court hinsichtlich 
der Kontrolle der Bundesgesetzgebungskompetenzen. Daher verwundert es nicht, daß seit den dreißiger Jahren bis zum Jahre 1995 kein einziges Kongreßgesetz wegen mangelnder Bundesgesetzgebungskompetenz aufgehoben wurde. Eine Entscheidung aus dem Jahre 1995, die ein Bundesgesetz für kompetenzwidrig erklärte, könnte nach Ansicht des Verfassers allerdings eine erneute Wende bei der Bestimmung der verfassungsgerichtlichen Kontrollbefugnisse in diesem Zusammenhang bedeuten.

Im dritten Teil befaßt sich Joswig mit der methodischen Herleitung der implied powersLehre (S. 142-159). Bezugnehmend auf die deutsche Staatsrechtswissenschaft zur methodischen Einordnung der sog. ungeschriebenen Bundeskompetenzen (Natur der Sache, Annexkompetenz, Kompetenz kraft Sachzusammenhangs) qualifiziert Joswig die implied powers-Lehre als Ergebnis einer Verfassungsauslegung, bei der Kompetenzen aus dem geschriebenen Verfassungsrecht durch einen Zweck-Mittel-Schluß als Methode der teleologischen Auslegung hergeleitet werden. Sei diese Auslegung nicht ausdrücklich von der Verfassung verboten, handele es sich um eine grundsätzlich zulässige Methode der Verfassungsinterpretation. Der Verfasser folgt damit der Einordnung der implied powers als in der Verfassung selbst wurzelnd und verdeutlicht die Abhängigkeit dieser Auslegungsmethode von dem konkreten Verfassungsverständnis mit einem Vergleich der Rechtsprechung des Supreme Court und des Bundesverfassungsgerichts. Nach dieser methodischen Einordnung der implied powers-Lehre trifft Joswig die Feststellung, daß die necessary and proper clause in Art. 1 sec. 8, cl. 18 der amerikanischen Verfassung nur deklaratorischen Charakter habe, da implied powers auch ohne eine derartige verfassungstextliche Verankerung definiert werden könnten. Die kurzen und bereits zum Verständnis der Rechtsprechungsübersicht im zweiten Teil wichtigen Ausführungen zur methodischen Einordnung enden mit einem Blick auf andere Anwendungsfelder der implied powers-Lehre in historischen Rechtsquellen sowie im Völker- und Europarecht, ohne allerdings auf sechs Seiten darauf wirklich eingehen zu können.

Im vierten und letzten Teil der Arbeit untersucht Joswig schließlich die Anwendungsbereiche der implied powers-Lehre bei der Exekutive durch den Präsidenten und der Judikative (S. 162-204). In der horizontalen Gewaltenteilung im Verhältnis zwischen Präsident und Kongreß stellt er eine überwiegend pragmatische Vorgehensweise des Supreme Court fest, der im Laufe der Zeit vier voneinander abweichende Grundkonzeptionen vertreten hat, ohne jemals ausdrücklich von einer früher vertretenen abzuweichen. Insgesamt sei die Tendenz festzustellen, die Lösung von Kompetenzkonflikten noch stärker als im Verhältnis zwischen Einzelstaaten und Gesamtstaat dem politischen Prozeß zu überlassen. In kritischer Auseinandersetzung mit der Rechtsprechung des Supreme Court spricht sich Joswig für eine verfassungsgerichtliche Kontrolle und eine differenzierende Anerkennung von implied powers des Präsidenten aus, je nachdem, ob die Maßnahme in den Kompetenzbereich einer anderen Gewalt eingreife oder nicht. Hinsichtlich der Anerkennung von implied powers der Judikative konstatiert Joswig lediglich einen Anwendungsfall von implied power bei einer richterrechtlich entwickelten Schadensersatzklage (implied constitutional rights of action), die persönlich gegen Bundesbedienstete in Fällen schwerer Grundrechts- 
verletzungen gerichtet werden kann, obwohl bundesrechtlich ein entsprechender Anspruch gegen diese Personengruppe gerade nicht vorgesehen ist.

In der Arbeit überwiegt eindeutig die Darstellung, die umfassend und dem Untersuchungsgegenstand entsprechend fallbezogen ist. Sofern der Verfasser vorsichtig eine eigene Wertung der Rechtsprechung des Supreme Court unternimmt, verbleibt diese auf einer recht abstrakten Ebene. Gerade im Rahmen der Darstellung der einzelnen Entscheidungen wäre es wünschenswert gewesen, die kritische Würdigung stärker in den konkreten Entscheidungszusammenhang einzubeziehen. Insgesamt handelt es sich bei Joswigs Untersuchung um eine gründliche und detaillierte Darstellung der implied powers-Lehre im US-amerikanischen Verfassungsrecht, die insbesondere dem mit den verfassungsrechtlichen Grundlagen der USA vertrauten Leser Gewinn bringt. Ihr besonderer Wert liegt darin, daß für den deutschen Leserkreis die Ursprünge der implied powers-Lehre im US-amerikanischen Verfassungsrecht und ihre Funktion in der dortigen Verfassungswirklichkeit in einer Weise vertieft werden, daß auch die wissenschaftliche Beschäftigung mit implied powers in anderem Zusammenhang, etwa im Völker- und Europarecht, daraus Nutzen ziehen kann.

Martin Düwel 